\title{
Tree species diversity of three Ghanaian reserves
}

\author{
Roberto Cazzolla Gatti (1), \\ Gaia Vaglio Laurin ${ }^{(2-3)}$ \\ Riccardo Valentini ${ }^{(2-3)}$
}

\begin{abstract}
Among tropical areas, Africa is considered to be poor in terms of biodiversity as compared with Amazon or South-East Asia, especially with respect to forest diversity. Despite this lower diversity, some African tropical zones, such as Ghana, harbour a plethora of species, particularly of trees. Unfortunately, as a result of anthropogenic impacts, biological diversity in West Africa dramatically decreased in the last decades, with very limited reference to evaluate the amount of the loss. Due to these growing pressure, a collection of relevant biodiversity information in this region seems to be urgent. We surveyed 127 temporary plots randomly distributed within 3 protected areas in Ghana and we collected data on tree $(\mathrm{dbh}>10 \mathrm{~cm})$ species richness and their abundances. We also performed $a$, and $B$ diversity analyses, and estimated the effective number of species, adopting various indices and approaches to provide further information on each assemblage. The main goals of this research were: (i) to provide a wide tree species database (abundance-based data), together with some biodiversity analyses; (ii) to estimate the sampling effort needed for next biodiversity surveys in the same and similar regions; and (iii) to calculate some indices useful to monitor the future of these protected areas both in terms of conservation and biodiversity research.
\end{abstract}

\section{Keywords: Ghana, Protected Areas, Forests, Database}

and monitor some of the most remote places on Earth (Danielsen et al. 2000); and (iii) the high level of complexity of tropical ecosystems (Bonn \& Gaston 2005). Among tropical areas, Africa is one of the least studied regions, especially in terms of rainforest diversity (Mayaux et al. 2013). Moreover, different protocols for surveying biodiversity in different regions of the world have been adopted, making difficult to compare studies and datasets (Gotelli \& Colwell 2001).

Timber extraction is the principal anthropogenic impact and the main cause of species extinction in terrestrial forest ecosystems (Harvey \& Pimentel 1996), and even selective logging has been questioned for its negative effects on both animal and plant diversity (Fredericksen \& Fredericksen 2002, Cazzolla Gatti et al. 2015, Vaglio Laurin et al. 2016). African tropical forests have been exploited and
(1) Biological Diversity and Ecology Laboratory, Bio-Clim-Land Centre of Excellence, Tomsk State University (TSU), 36 Lenin Prospekt, Tomsk, 634050 (Russia); (2) Impacts of Agriculture, Forests and Ecosystem Services Division, Euro-Mediterranean Center on Climate Change (IAFES-CMCC), v. Pacinotti 5, I-01100 Viterbo (Italy); (3) Department for Innovation in Biological, Agro-Food and Forest Systems (DIBAF), University of Tuscia, I-01100 Viterbo (Italy)

@ Roberto Cazzolla Gatti (robertocgatti@gmail.com)

Received: Mar 14, 2016 - Accepted: Jan 03, 2017

Citation: Cazzolla Gatti R, Vaglio Laurin G, Valentini R (2017). Tree species diversity of three Ghanaian reserves. iForest 10: 362-368. - doi: 10.3832/ifor2056-010 [online 2017-03-07]

Communicated by: Marco Ferretti severely disturbed by anthropogenic activities for many centuries (Valentini et al. 2014). In particular, West African forests were overexploited for timber exportation and agricultural development during the European colonization (Barnes 1990). The result is a fragmented landscape from Guinea to Nigeria, composed by patches of forest interspersed in a rural environment (Vaglio Laurin et al. 2013). Consequently, biological diversity dramatically decreased in the last few decades, with very limited reference to evaluate the amount of the loss (Dupouey et al. 2002). Due to the growing anthropogenic pressure in the West African region, it seems urgent to collect relevant biodiversity information. These data could be used as a baseline for future studies aimed at the analysis of environmental changes (Mace \& Baillie 2007).

Tree diversity is often considered as a good proxy to estimate diversity of other taxonomic groups (Gentry 1988). Because tree diversity can be measured at different levels, in this research we evaluated three biodiversity components, in order to provide an exhaustive biodiversity information: (i) a diversity, as the mean species diversity of each study site; (ii) $\beta$ diversity, as both the difference in diversity among sites and among plots at each site; and (iii) effective species number, as the overall species richness in each study site.

On a theoretical basis, at the spatial scale of a hectare or less, evolutionary-biogeographical influences and variation in physical factors are constrained to a minimum (Levey et al. 2002). For instance, one such set of interactions is described by the Jan- 
zen-Connell mechanism, which proposes that the probability of survival of a seed or the successful establishment of a seedling increases with distance from its parent tree. Seeds that fall relatively far from the parent increase survival, as they grow distant from predators, herbivores and/or pathogens which affect mother plants (Janzen 1970, Connell 1971). In micro-scale, Janzen-Connell's idea could explain the high tree diversity observed within small plots in tropical areas, but further basic and detailed data on tropical tree diversity are needed to test this and other hypotheses.

The objectives of this study are: (i) to provide baseline information on tree diversity in the form of a wide database for the three protected areas surveyed in Ghana; (ii) to estimate the minimum sampling effort needed to collect the effective number of species in other comparative studies in the same or ecologically similar protected areas; (iii) to provide multiple biodiversity measures able to summarize these data.

In order to achieve these targets, we surveyed three protected areas in Ghana where we collected data at species level, performing $\alpha$ and $\beta$ diversity analyses. We also estimated the effective number of species and the minimum sampling area, employing various indices and measures.

\section{Materials and methods}

Study sites

Bia Conservation Area (CA) is located in the Juabeso-Bia District in southwest Ghana, close to the border with Ivory Coast. This CA covers approximately 306

Tab. 1 - Sampling effort and a diversity indices for the three study areas.

\begin{tabular}{lccc}
\hline Study site & $\begin{array}{c}\text { Total no. } \\
\text { of plots }\end{array}$ & $\begin{array}{c}\text { Area sampled } \\
\text { (ha) }\end{array}$ & $\begin{array}{c}\text { No. of } \\
\text { trees }\end{array}$ \\
\hline Ankasa CA & 34 & 1.70 & 841 \\
Dadieso FR & 46 & 1.84 & 402 \\
Bia CA & 47 & 1.88 & 625 \\
\hline Index & Ankasa CA & Bia CA & Dadieso FR \\
\hline Number of Species (S) & 147 & 128 & 120 \\
Number of Individuals (N) & 841 & 625 & 402 \\
Margalef's (Richness) & 21.68 & 19.73 & 19.85 \\
Relative dominance & 0.081 & 0.077 & 0.060 \\
Singletons & 54 & 51 & 48 \\
Doubletons & 22 & 18 & 22 \\
Uniques & 57 & 54 & 55 \\
Alpha-Fisher & 51.55 & 48.74 & 57.91 \\
Alpha-Fisher SD & 2.94 & 3.15 & 4.57 \\
ACE & 205.83 & 192.87 & 169.26 \\
Chao 1 & 213.19 & 200.13 & 172.23 \\
Shannon & 4.23 & 4.26 & 4.35 \\
Shannon Evenness & 0.85 & 0.88 & 0.91 \\
Simpson (1/D) & 39.15 & 44.68 & 51.47 \\
Simpson Evenness & 0.27 & 0.35 & 0.43 \\
\hline Guild Pioneer \% & 7.53 & 18.97 & 18.33 \\
Guild Shadow tolerant \% & 21.23 & 35.34 & 35.83 \\
Guild NPLD \% & 61.64 & 43.10 & 41.67 \\
Guild Swamp \% & 2.85 & 2.50 \\
Not classified \% & 2.74 & 0.00 & 1.67 \\
\hline
\end{tabular}

\section{Biodiversity data sampling}

Field data were collected in the abovementioned study sites between 2011 and 2013 in the framework of different research projects, retaining for the present analysis only variables common to all the surveys and collected with same protocols. Identification of tree species was conducted with the help of the same botanists in all areas (Appendix 1).

Rectangular (20×25 m in Ankasa CA) or square $(20 \times 20 \mathrm{~m}$ in Bia NA and Dadieso FR) spatially separated plots were arranged in each study site with a random placement protocol (Magurran 2013, Messina et al. 2016), within a circular area of $1 \mathrm{~km}$ of radius, established in the geometrical center of each reserve.

For all study sites we chosen a minimum common threshold of diameter at breast height ( $\mathrm{dbh}$ ) of $10 \mathrm{~cm}$, as this encompassed the main diversity of the analysed tropical forest, and $5<\mathrm{dbh}<10 \mathrm{~cm}$ did not add relevant information to the data (Cazzolla Gatti et al. 2015). All trees with $\mathrm{dbh}>10 \mathrm{~cm}$ were identified at species level and their abundances were assessed (Tab. S1 in Supplementary material). Tab. 1 illustrates the number of field plots, the area sampled and the number of recorded trees. The identification of the species functional guilds was conducted following the classification developed by Hawthorne (1995) who identified pioneer, non-pioneer light demanding (NPLD), shade tolerant, and swamp species.

\section{Calculation and statistics}

To perform $\alpha$ and $\beta$ species diversity analyses we used the software package EstimateS (Colwell 2013). We computed a variety of biodiversity statistics, including rarefaction and accumulation curves, estimators of effective species number, diversity indices, and similarity measures. Standard error buffers and bootstraps techniques were employed when appropriate.

We produced species-area curves (SACs) as accumulation curves for comparison purposes, due to their wide use in botanical research; these curves allow comparison of sites and provide information on the minimum sampling efforts needed to capture the local diversity (Magurran 2013). SACs were calculated for each study site by plotting sampling area $(A)$ increments (in terms of accumulating number of plots) against number of species sampled $(S)$, with 100 randomization of sample units to obtain a smoothed curve. A log-log transformation of SACs was utilized to derive $c$ and $z$ parameters of the equation (eqn. 1):

$$
S=c A^{z}
$$

or in log scale (eqn. 2):

$$
\log S=c+z \log A
$$

We estimated the species-area saturation value for each area adopting the Chao1 and 
the Abundance-Coverage Estimator (ACE) indexes (Magurran 2013). These measures can provide an approximation of the expected tree diversity in each area, and can also be considered as a proxy of the effective number of species (Magurran 2013). SACs are sources of reliable information to adequately sample the assemblages (Gotelli \& Colwell 2001).

We derived Coleman's rarefaction curves to evaluate the number of species at the same abundances level, thus comparing the richness of three study areas characterized by different sampling efforts. We also plotted the frequency-distribution graph to analyse dominance/evenness patterns, and the rank-abundance (Whittaker's) plot to evaluate the total richness, the abundances distribution, and to evidence differences in evenness among the assemblages. We tested the significance of the abundances distribution patterns by the Kolmogorov-Smirnov statistical test (here at 95\% confidence level).

Together with the Whittaker's plots we show the Empirical Cumulative Distribution Function (ECDF) graph, which allows a better discrimination of different assemblages obtained by rescaling the ranks according to the richness (Magurran 2013).

$\beta$-diversity analysis were performed computing the Marczewski-Steinhaus index to understand the dissimilarity between pairs of sites (i.e., $\beta$-diversity among sites). Because this measure considers only species presence and not species abundances data, it is not influenced by the dominance of certain species in the assemblage (Magurran 2013). Thus, we also computed the complement of Morisita-Horn abundancebased $\beta$-diversity measure $\left(1-\mathrm{C}_{\mathrm{MH}}\right)$ to understand which influence the abundance of species can have on similarity. $\beta$-diversity was also calculated among plots of each study site ( $\beta$-diversity within site) to evaluate "internal" small-scale diversity patterns.

The effective number of species was estimated at study area level by the Chao1 index and the Abundance-Coverage Estimator (ACE - Magurran 2013).

\section{Results}

\section{Richness, $\alpha$ diversity and guild} composition

The list of species and their abundances in each study site are reported in Tab. S1 (Supplementary material). The results illustrated in Tab. 1 indicates that Bia and Dadieso are very similar in a-diversity and guilds' percentage composition, while Ankasa differs from both. Although the sampling effort in Ankasa was the smallest, species richness (147) of this CA was higher by 19 and 27 species, respectively, than Bia and Dadieso. Margalef, ACE and Chao1 indices of Ankasa were also the highest.

The number of singletons recorded in Ankasa was also greater than the other two areas, with 54 singletons out of 147

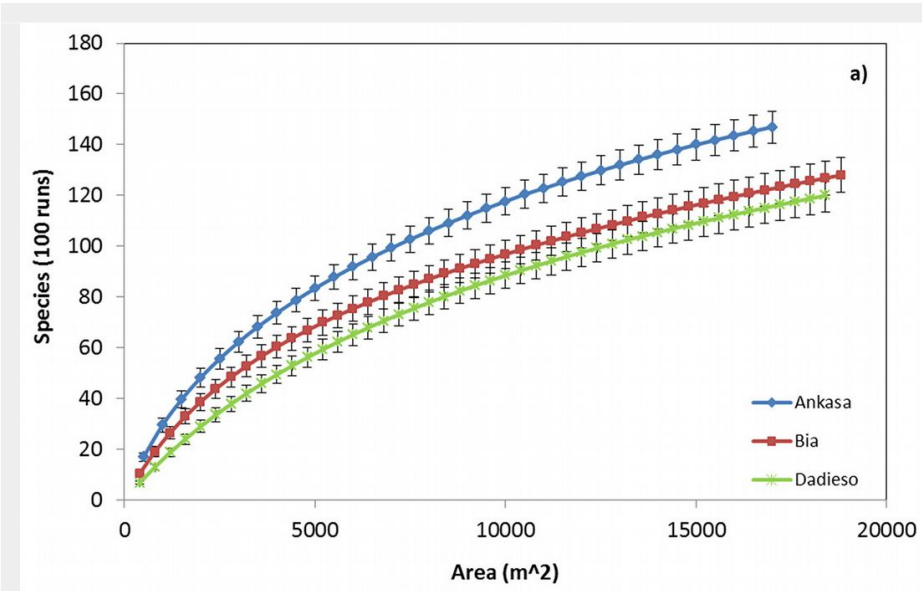

Fig. 1 - Speciesarea curves (a), Coleman's rarefaction curves (b) and frequency-abundance distributions (c) for the three study areas. Error bars represent the standard deviation.
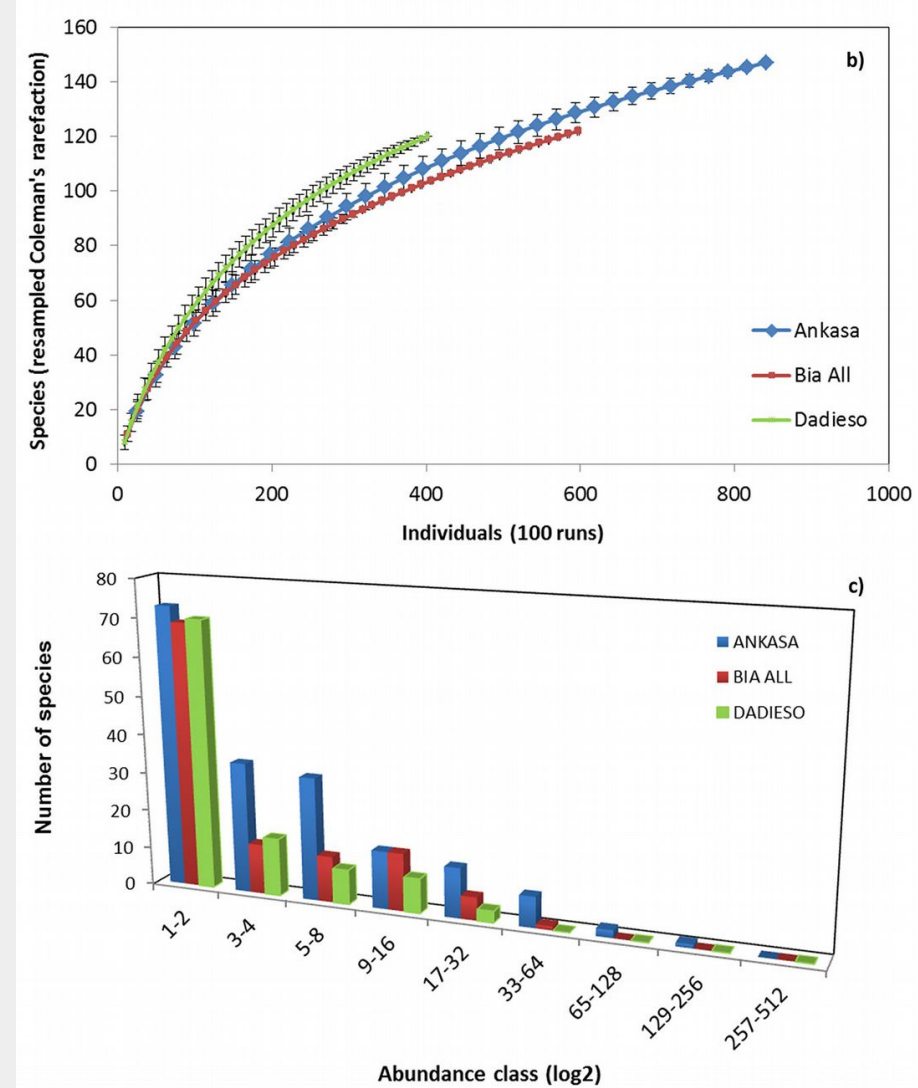

species sampled in this area. The guilds percentages analysis shows that Ankasa was the only site characterized by a dominant guild reaching $60 \%$, with almost $20 \%$ of shadow tolerant species and a low amount $(<10 \%)$ of pioneer species. Bia and Dadieso, instead, had a lower percentage $(\approx 40 \%)$ of dominant species and a similar level of shadow tolerant species, while pioneers ranged between $15-20 \%$. The swamp guild was more represented in Ankasa $(\approx 7 \%)$ than in the other two sites (2.50\% and $2.59 \%$ in Dadieso and Bia, respectively).

\section{Accumulation and rarefaction curves,} and abundance-frequency distribution

Ankasa showed the upper SAC curve, even considering the standard deviation buffers (Fig. 1a). The minimum sampling area needed to achieve a representative collection of species at each site is presented in Tab. 2. This estimation indicates minimum values of about 3 ha in each of the three sites.

Coleman's rarefaction curves, obtained after rarefaction at the minimum sample size (equal to 384 individuals), indicated that Dadieso was the richest site, while Ankasa was intermediate and Bia was the poorest (Fig. 1b). The frequency-distribution plot (Fig. 1C) evidenced the differences among the study areas. Ankasa has well represented frequencies in 3-4, 5-8 and the following abundance classes, which slightly decline. Dadieso and Bia showed lower frequencies in classes other than 1-2 and zero frequency at abundance value higher than 33-64 individuals per species. Moreover, Bia showed a bimodal distribution that peaks at 1-2 and 9-16 classes of abundance.

Species-abundance curves, rankabundance plots, and ECDF

Cumulative abundance of species was 
Tab. 2 - Minimum sampling area estimation.

\begin{tabular}{lcccccccc}
\hline Study site & $\log c$ & $\boldsymbol{c}$ & $\boldsymbol{z}$ & $\begin{array}{c}\mathbf{1 / z} \\
(\mathrm{Rad} \boldsymbol{z})\end{array}$ & Chao 1 & $\begin{array}{c}\text { Area } \\
\text { saturation } \\
\text { Chao 1 (ha) }\end{array}$ & $\begin{array}{c}\text { Area } \\
\text { ACE }\end{array}$ & $\begin{array}{c}\text { saturation } \\
\text { ACE (ha) }\end{array}$ \\
\hline Ankasa CA & -0.225 & 0.595 & 0.57 & 1.754 & 213 & 3.02 & 206 & 2.84 \\
Dadieso FR & -0.895 & 0.127 & 0.71 & 1.408 & 195 & 3.07 & 188 & 2.92 \\
Bia CA & -0.374 & 0.422 & 0.59 & 1.694 & 200 & 3.43 & 193 & 3.22 \\
\hline
\end{tabular}

Fig. 2 - Abundance-species curve (a), rankabundance plots (b) and ECDF (c) for the three study are-sites. $\mathrm{n} / \mathrm{N}$ is the relative abundance.
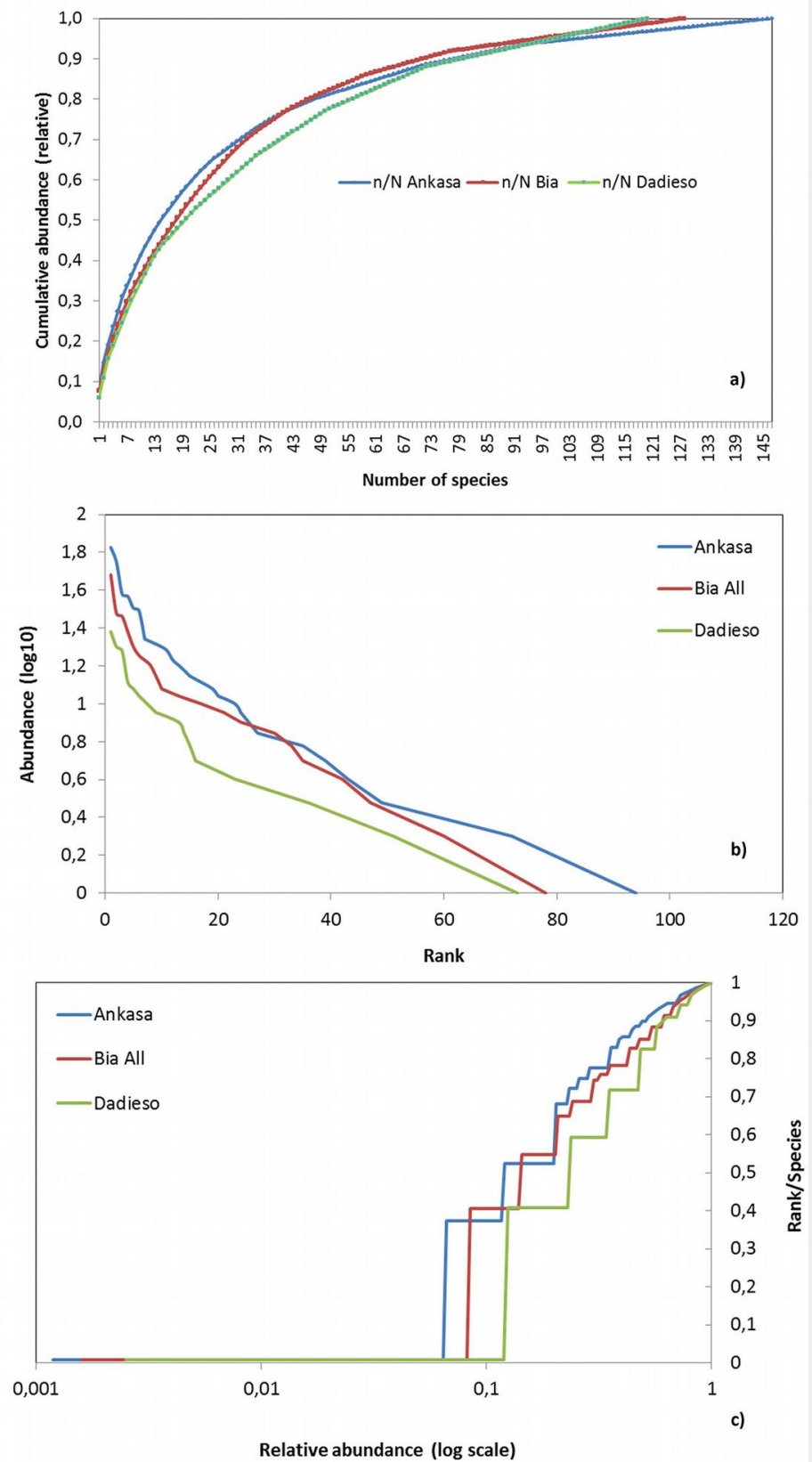

Tab. 3 - Among (a) and within (b) study areas $\beta$ diversity. (SD): standard deviation.

\begin{tabular}{|c|c|c|c|c|}
\hline \multirow{2}{*}{ (a) } & \multicolumn{2}{|c|}{ Marczewski-Steinhaus } & \multicolumn{2}{|c|}{ Morisita-Horn $\left(1-\mathrm{C}_{\mathrm{MH}}\right)$} \\
\hline & Ankasa & Bia & Ankasa & Bia \\
\hline Dadieso & 0.77 & 0.59 & 0.81 & 0.51 \\
\hline Ankasa & - & 0.79 & - & 0.87 \\
\hline (b) & \multicolumn{2}{|c|}{ Mean \pm SD } & \multicolumn{2}{|c|}{ Mean \pm SD } \\
\hline Ankasa & \multicolumn{2}{|c|}{$0.86 \pm 0.06$} & \multicolumn{2}{|c|}{$0.73 \pm 0.16$} \\
\hline Bia & \multicolumn{2}{|c|}{$0.91 \pm 0.08$} & \multicolumn{2}{|c|}{$0.84 \pm 0.16$} \\
\hline Dadieso & \multicolumn{2}{|c|}{$0.95 \pm 0.07$} & \multicolumn{2}{|c|}{$0.93 \pm 0.12$} \\
\hline
\end{tabular}

plotted against the species ranked according to their abundances (Fig. 2a). The $x$ axes level of the inflection point indicates the quota of dominant species.

Fig. 2b shows the Whittaker's plots of the study areas. Ankasa has a lightly steeper curve than Bia and Dadieso. Being the richness estimated from the $x$-axes point where the curves end, this diagram remarks the increasing richness from Dadieso, to Bia and then to Ankasa. The distributions of abundances were not significantly different $(p>0.05)$ for any CA pair after two-samples Kolmogorov-Smirnov test (Dadieso-Ankasa $\mathrm{D}_{[120,147]}=0.09$; Ankasa-Bia $\mathrm{D}_{[147,128]}=0.05$; Bia-Dadieso $\left.\mathrm{D}_{[128,120]}=0.08\right)$.

The ECDF for the three areas is shown in Fig. 2c. Plotting the assemblages with this function, Ankasa curve was still higher than those of Bia and Dadieso. Ankasa curve was also steeper, which means that it is less even than the other two CAs. The lower left part of the curves show that Ankasa and Bia accounted for more rare species than Dadieso, which is upper positioned.

\section{Effective number of species and $\beta$ diversity}

The ACE and Chao1 indices (here considered as proxy of the effective number of species) were the highest for Ankasa, Bia values were intermediate and those of Dadieso the lowest (Tab. 1). The minimum sampling area estimates, which is based on those measures, showed a value of about 3 ha to collect the expected number of species for each of the study sites (Tab. 2).

Marczewski-Steinhaus and complement Morisita-Horn indices calculated among paired study areas shows that Bia-Ankasa pair was characterized by the highest $\beta$ diversity, while Dadieso-Bia pair by the lowest, and Ankasa-Dadieso stands in the middle (Tab. 3a). The same indexes calculated within each area shows that the highest internal $\beta$ diversity was found in Dadieso, while the lowest was in Ankasa, with intermediate values in Bia (Tab. 3b).

\section{Discussion}

We provided tree diversity data for three protected areas in Ghana, including information on the abundances of species, in a region where field data are sometimes scarce and relatively difficult to collect. The evaluation of different indices and measures allowed to preliminary characterize part of the diversity of the areas, also providing indication on the minimum sampling 
effort needed to collect the effective number of species in these and similar forests, which could encompass and include the species missed by our pilot study.

Since only a portion of the reserves was considered, results cannot be extrapolated to the entire areas, but this study should be considered as a pilot report in order to provide background information for future research. Moreover, sample plots of different shape and size could have caused possible bias. For instance, Ankasa CA, with the largest and rectangular sample plots, was also the richest, and this may be at least partially due to different shape and size of the plots.

Most of the indices and graphs indicate that Ankasa is the richest site among those surveyed, although the sampled area is the smallest among sites. This confirms what was observed by Martin (1991) that this Conservation Area has the highest tree richness level found in West Africa. While most of the tested indexes, measures and curves have higher values in Ankasa (e.g., Margalef, ACE, Chao1, rare species, N, S, SAC curve, ECDF), the Shannon and Simpson diversity measures for this area are, instead, the lowest among the three sites, and Alpha-Fisher shows an intermediate value. This result was expected as these diversity measures are strongly influenced by the abundances of dominant species: the presence of few dominant and many rare species produces a low evenness. This is also evidenced in Ankasa by the speciesabundance curve. Coleman's rarefaction curves show Ankasa in an intermediate position, between Dadieso and Bia. We argue that the random removal of almost half of the individuals through rarefaction procedure (from the 841 collected in Ankasa to the rarefaction value of 384.52 ) proportionally reduced the richness of uneven sites more than the richness of even ones. As documented in other tropical and complex ecosystems (Stirling \& Wilsey 2001, Cazzolla Gatti et al. 2015), richness measures do not always match the diversity indexes, and it is advisable to carefully consider both richness and diversity measures. The occurrence of swamps in Ankasa creates a diversification of this ecosystem, with a consequent addiction of species in the related guild, which also contributes to the high richness of this site. Moreover, the total number of species we collected in Ankasa is higher than those recorded by other studies in the same area (Asase et al. 2012).

A major difference among the study sites is the presence of pioneer species. Even if untouched forest can also harbour some of them, pioneers are considered as an indicator of disturbance and degradation (Kohyama 1993). Ankasa showed a much lower percentage of pioneers than Bia and Dadieso and this is a confirmation of the low disturbance occurred in this area, which also allowed the development of such a rich site. An additional evidence of the low disturbance in Ankasa results from the comparison of the abundance-frequency distributions and Whittaker's plots of the three study areas.

Nevertheless, due to sampling limitations and the consequent lack of a full collection of very rare species, in each protected area the left side of abundances-frequency distributions is hidden (Preston 1948).

The Kolmogorov-Smirnov test evidenced no significant difference in the distributions of the abundances of species among the three sites. It follows that, although different in vegetation type, logging history and climatic zone, the species composition of these protected areas can be easily compared in terms of their diversity. This could be the reason why we obtained the same minimum sampling area of about 3 hectares in every study areas. This value is much higher than what was previously adopted in other studies on tree diversity of the region (Asase et al. 2012, Appiah 2013). We estimate that the mean effective number of species (Colwell \& Coddington 1994) that can be collected in a minimum sampling areas of 3 hectares in each site is about 200 (SChao1 \pm SD = $202.67 \pm 9.29$; SACE $\pm S D=195.67 \pm 9.29)$. If considered together, a total of 250 species and 1868 individuals were recorded within the three protected areas (see Tab. S1 in Supplementary material for the plot-based abundance data).

We looked at SACs to locate the number of species per hectare we collected in our study sites, which is in the range of 88-118. Then we compared these values to some other tropical forest sites in the Amazon and in tropical Asia, where tree $(\mathrm{dbh} \geq 10$ $\mathrm{cm}$ ) species number per hectare reached values of 300 (Valencia et al. 1994) and 200 (Parmentier et al. 2007), respectively.

As mentioned before, the results of this study are preliminary and should be considered with caution, as only a small portion of the sites have been sampled and our data could not be representative of the whole reserves. Nonetheless, we found that all the three protected areas we surveyed are richer than other other African tropical forests, where the number of tree species with $\mathrm{dbh} \geq 10 \mathrm{~cm}$ per hectare was no more than 73 (Whitmore \& Sidiyasa 1986). A more recent study (Phillips et al. 1994) analysed different tropical forests, reporting values of 86-92 tree species per hectare in Ghana and 56 in Uganda. This confirms that Bia and Dadieso forests are within the range of other Ghanaian forests as for species number, but still richer than other tropical African sites. Contrastingly, Ankasa is always well above the richness of all Ghanaian and most of African forests.

To better evaluate richness similarities and differences, in order to reduce the bias due to the area effect, we considered the number of species per 500 individuals reported in Phillips et al. (1994) and compared it with our values derived from Coleman's rarefaction curves. In the previous study 82-89 species/500 individuals were collected in Ghana, while applying rarefaction to the same number of individuals on our data we found 114-120 species/500 individuals in all the three sites. Unexpectedly, these values are in line with most of Amazonian sites (Costa Rica, Venezuela, Ecuador and Brazil, in particular) reported by Phillips et al. (1994), with the only exception of Peru. South-east Asian forests show always higher numbers of tree species. This preliminary comparison suggests that the tree diversity of African forests might be reconsidered, as it could be comparable with that of some South American sites when individuals, instead of area, are taken into account. This underlines that tropical forest diversity is not always a matter of quantity (i.e., number of species in area units), but sometimes an issue of quality (i.e., how individuals distribute in space and among species - Cazzolla Gatti 2011, Cazzolla Gatti 2016d).

The within plots $\beta$-diversity of Dadieso is much higher than those of the other two forests. This means that this forest reserve has a great spatial diversity of species richness, which usually reflects the heterogeneity of the environment. Indeed, Dadieso is a transitional forest that preserves both moist evergreen and wet evergreen species, including species typical of swamps.

The highest $\beta$-diversity between sites was found for the Ankasa-Bia pair and this diversity evidences the climatic and ecological differences (wet vs. moist evergreen) between these forest types. Dadieso has intermediate values when paired with both Ankasa and Bia, reflecting the mixed vegetation type that characterizes this reserve.

In conclusion, the data and indices we provide could be useful to address next studies on these and similar sites, and can be considered as a reference in order to monitor the future of the surveyed protected areas.

\section{Acknowledgments}

We acknowledge the ERC grant Africa GHG \#247349 for providing research funding. We are very grateful to the Ghana Forestry Commission staff, the botanist $\mathrm{Mr}$ Ntim Gyakari, Justice Mensah, and all the collaborators who supported the field activities in Ghana.

\section{References}

Appiah M (2013). Tree population inventory, diversity and degradation analysis of a tropical dry deciduous forest in Afram Plains, Ghana. Forest Ecology and Management 295: 145-154. doi: 10.1016/j.foreco.2013.01.023

Asase A, Asitoakor BK, Ekpe PK (2012). Linkages between tree diversity and carbon stocks in unlogged and logged West African tropical forests. International Journal of Biodiversity Science, Ecosystem Services and Management 8 (3): 217-230. - doi: 10.1080/21513732.2012.707 152

Barnes RFW (1990). Deforestation trends in 
tropical Africa. African Journal of Ecology 28 (3): 161-173. - doi: 10.1111/j.1365-2028.1990.tbo11 50.x

Battipaglia G, Zalloni E, Castaldi S, Marzaioli F, Cazzolla Gatti R, Lasserre B, Tognetti R, Marchetti M, Valentini R (2015). Long tree-ring chronologies provide evidence of recent tree growth decrease in a central African tropical forest. PloS one 10 (3): e0120962. - doi: 10.1371/ journal.pone.0120962

Bonn A, Gaston KJ (2005). Capturing biodiversity: selecting priority areas for conservation using different criteria. Biodiversity and Conservation 14 (5): 1083-1100. - doi: 10.1007/s10531004-8410-6

Cazzolla Gatti R (2011). Evolution is a cooperative process: the biodiversity-related niches differentiation theory (BNDT) can explain why. Theoretical Biology Forum 104 (1): 35-43.

Cazzolla Gatti R, Castaldi S, Lindsell JA, Coomes DA, Marchetti M, Maesano M, Di Paola A, Paparella F, Valentini R (2015). The impact of selective logging and clearcutting on forest structure, tree diversity and above-ground biomass of African tropical forests. Ecological Research 30 (1): 119-132. - doi: 10.1007/s11284014-1217-3

Cazzolla Gatti R (2016a). The fractal nature of the latitudinal biodiversity gradient. Biologia 71 (6): 669-672. - doi: 10.1515/biolog-2016-0077 Cazzolla Gatti R (2016b). Trends in human development and environmental protection. International Journal of Environmental Studies 73 (2): 268-276. - doi: 10.1080/00207233.2016.1148447 Cazzolla Gatti R (2016c). Freshwater biodiversity: A review of local and global threats. International Journal of Environmental Studies 73 (6): 887-904. - doi: 10.1080/00207233.2016.1204133 Cazzolla Gatti R (2016d). A conceptual model of new hypothesis on the evolution of biodiversity. Biologia 71 (3): 343-351. - doi: 10.1515/biol og-2016-0032

Chazdon RL, Colwell RK, Denslow JS, Guariguata MR (1998). Statistical methods for estimating species richness of woody regeneration in primary and secondary rain forests of NE Costa Rica. In: "Forest biodiversity research, monitoring and modeling: Conceptual background and Old World case studies" (Dallmeier F, Comiskey JA eds). Parthenon Publishing, Paris, pp. 285309. [online] URL: http://www.cifor.org/libra ry/456/

Colwell RK (2013). EstimateS: statistical estimation of species richness and shared species from samples. Version 9. User's Guide and application. Web site. [online] URL: http:// purl.oclc.org/estimates

Colwell RK, Coddington JA (1994). Estimating terrestrial biodiversity through extrapolation. Philosophical Transactions: Biological Sciences 345: 101-118. - doi: 10.1098/rstb.1994.0091

Connell JH (1971). On the role of natural enemies in preventing competitive exclusion in some marine animals and in rain forest trees. Dynamics of populations 298: 312.

Connell JH (1978). Diversity in tropical rain forests and coral reefs. Science 199 (4335): 13021310. - doi: 10.1126/science.199.4335.1302

Danielsen F, Balete DS, Poulsen MK, Enghoff M, Nozawa CM, Jensen AE (2000). A simple system for monitoring biodiversity in protected areas of a developing country. Biodiversity and Conservation 9 (12): 1671-1705. - doi: 10.1023/ A:1026505324342

Dupouey JL, Dambrine E, Laffite JD, Moares C (2002). Irreversible impact of past land use on forest soils and biodiversity. Ecology 83 (11): 2978-2984. - doi: 10.1890/0012-9658(2002)083 [2978:IIOPLU]2.0.CO;2

Fredericksen NJ, Fredericksen TS (2002). Terrestrial wildlife responses to logging and fire in a Bolivian tropical humid forest. Biodiversity and Conservation 11 (1): 27-38. - doi: 10.1023/A:1014 065510554

Gentry AH (1988). Changes in plant community diversity and floristic composition on environmental and geographic gradients. Annals of the Missouri Botanical Garden 75: 1-34. - doi: 10.230 7/2399464

Gotelli NJ, Colwell RK (2001). Quantifying biodiversity: procedures and pitfalls in the measurement and comparison of species richness. Ecology letters 4 (4): 379-391. - doi: 10.1046/j.14610248.2001.00230.x

Haines-Young R, Potschin M (2010). The links between biodiversity, ecosystem services and human well-being. Ecosystem Ecology: a new synthesis, Cambridge University Press, Cambridge, UK, pp. 110-139. - doi: 10.1017/CBO97805117504 58

Hall JB, Swaine MD (1981). Distribution and ecology of vascular plants in a tropical rain forest: forest vegetation in Ghana. The Hague, Boston, MA, USA, pp. 383.

Harvey CA, Pimentel D (1996). Effects of soil and wood depletion on biodiversity. Biodiversity and Conservation 5 (9): 1121-1130. - doi: 10.1007/ BFo0052721

Hawthorne WD (1995). Ecological profiles of Ghanaian forest trees. Tropical Forestry Papers, No. 29, vi, pp. 345. [online] URL: http://www. cabdirect.org/cabdirect/abstract/19950616640 Janzen DH (1970). Herbivores and the number of tree species in tropical forests. The American Naturalist 104 (940): 501-528. - doi: 10.1086/282 687

Jones C, Lawton JH (2012). Linking species and ecosystems. Chapman and Hall, New York, USA, pp. 387.

Kohyama T (1993). Size-structured tree populations in gap-dynamic forest - the forest architecture hypothesis for the stable coexistence of species. The Journal of Ecology 81 (1): 131. doi: $10.2307 / 2261230$

Lenton TM, Lovelock JE (2000). Daisyworld is Darwinian: constraints on adaptation are important for planetary self-regulation. Journal of Theoretical Biology 206 (1): 109-114. - doi: 10.100 6/jtbi.2000.2105

Levey DJ, Silva WR, Galetti M (2002). Seed dispersal and frugivory: ecology, evolution and conservation. CABI Publishing, Oxon, UK, pp. 511.

Mace GM, Baillie JE (2007). The 2010 biodiversity indicators: challenges for science and policy. Conservation Biology 21 (6): 1406-1413. - doi: 10.1111/j.1523-1739.2007.00830.x

Magurran AE (2013). Measuring biological diversity. John Wiley and Sons, Oxford, UK, pp. 264. [online] URL: http://books.google.com/books? id=fljsaxmL_S8C

Martin C (1991). The rainforests of West Africa.
Ecology-threats-conservation. Springer Verlag, Berlin, Germany, pp. 235. [online] URL: http:// www.cabdirect.org/cabdirect/abstract/1991065 0813

Mayaux P, Pekel JF, Desclee B, Donnay F, Lupi A, Achard F, Clerici M, Bodart C, Brink A, Nasi R, Belward A (2013). State and evolution of the African rainforests between 1990 and 2010. Philosophical Transactions of the Royal Society B: Biological Sciences 368 (1625): 20120300. doi: $10.1098 /$ rstb.2012.0300

Messina G, Cazzolla Gatti R, Droutsa A, Barchitta M, Pezzino E, Agodi A, Lombardo BM (2016). A sampling optimization analysis of soil-bugs diversity (Crustacea, Isopoda, Oniscidea). Ecology and Evolution 6 (1): 191-201. - doi: 10.1002/e ce3.1765

Parmentier I, Malhi Y, Senterre B, Whittaker RJ, Alonso A, Balinga MP, Bakayoko A, Bongers F, Chatelain C, Comiskey JA, Cortay R, Kamdem $M D$, Doucet JL, Gautier L, Hawthorne WD, Issembe YA, Kouamé FN, Kouka LA, Leal ME, Lejoly J, Lewis SL, Nusbaumer L, Parren MP, Peh KS, Phillips OL, Sheil D, SonkÉ B, Sosef MS, Sunderland TC, Stropp J, Ter Steege H, Swaine MD, Tchouto MG, Gemerden BS, Van Valkenburg JL, Wöll H (2007). The odd man out? Might climate explain the lower tree diversity of African rain forests relative to Amazonian rain forests? Journal of Ecology 95 (5): 1058-1071. doi: 10.1111/j.1365-2745.2007.01273.x

Phillips OL, Hall P, Gentry AH, Sawyer SA, Vasquez R (1994). Dynamics and species richness of tropical rain forests. Proceedings of the National Academy of Sciences 91 (7): 28052809. - doi: 10.1073/pnas.91.7.2805

Preston FW (1948). The commonness, and rarity, of species. Ecology 29 (3): 254-283. - doi: 10.23 07/1930989

Stirling G, Wilsey B (2001). Empirical relationships between species richness, evenness, and proportional diversity. The American Naturalist 158 (3): 286-299. - doi: 10.1086/321317

Vaglio Laurin G, Liesenberg V, Chen Q, Guerriero L, Del Frate F, Bartolini A, Coomes D, Wilebore B, Lindsell J, Valentini R (2013). Optical and SAR sensor synergies for forest and land cover mapping in a tropical site in West Africa. International Journal of Applied Earth Observation and Geoinformation 21: 7-16. - doi: 10.1016/j.jag.20 12.08.002

Vaglio Laurin G, Hawthorne WD, Chiti T, Di Paola A, Cazzolla Gatti R, Marconi S, Noce S, Grieco E, Pirotti F, Valentini R (2016). Does degradation from selective logging and illegal activities differently impact forest resources? A case study in Ghana. iForest 9: 354-362. - doi: 10.3832/ifor 1779-008

Valencia R, Balslev H, Miño GPY (1994). High tree alpha-diversity in Amazonian Ecuador. Biodiversity and Conservation 3 (1): 21-28. - doi: 10.1007/ BFo0115330

Valentini R, Arneth A, Bombelli A, Castaldi S, Cazzolla Gatti R, Chevallier F, Ciais P, Grieco E, Hartmann J, Henry $M$, Houghton RA, Jung $M$, Kutsch WL, Malhi Y, Mayorga E, Merbold L, Murray-Tortarolo G, Papale D, Peylin P, Poulter $B$, Raymond PA, Santini M, Sitch S, Vaglio Laurin G, Van Der Werf GR, Williams CA, Scholes RJ (2014). A full greenhouse gases budget of Africa: synthesis, uncertainties, and vulnerabili- 
ties. Biogeosciences 11: 381-407. - doi: 10.5194/ Supplementary Material bg-11-381-2014

Whitmore TC, Sidiyasa K (1986). Composition and structure of a lowland rain forest at Toraut, northern Sulawesi. Kew Bulletin 41 (3): 747. doi: $10.2307 / 4103127$
Appendix 1 - Study site supplementary information.

Appendix 2 - Supplementary methods.

Appendix 3 - Supplementary conclusions.
Fig. S1 - The three study areas in Ghana, West Africa.

Tab. S1 - Species list and species abundances in each study site.

Link: Cazzolla_2056@supplo01.pdf 